\title{
Hands on GINGER: Seismic Wave measurement
}

\author{
Federico Ferraro* \\ Università degli Studi di Genova, Dipartimento di Fisica, Genova, Italy \\ INFN, Sezione di Genova, Genova, Italy \\ E-mail: federico.ferraro@ge.infn.it
}

\section{Angela Di Virgilio}

INFN, Sezione di Pisa, Pisa, Italy

E-mail: angela.divirgilio@pi.infn.it

GINGER (Gyroscopes IN GEneral Relativity) will be a 3-D array of mutually orthogonal ring lasers able to measure the general relativistic effects due to the rotation of the Earth (LenseThirring effect).

The development of highly sensitive ring laser gyroscopes gives as well the possibility to accurately monitor the rotational ground motions on Earth.

The GP2 ring laser, a prototype developed to study how to keep constant at the level of 1 part in $10^{10}$ the scale factor of the ring lasers in the GINGER array, has been recently designed and realized and some of its data have been analyzed for geophysical studies. The signal has been extracted from the interferogram raw data and the seismic wave contribution has been obtained by subtracting the contribution due to the Earth rotation.

Gran Sasso Summer Institute 2014 Hands-On Experimental Underground Physics at LNGS - GSSI14, 22 September - 03 October 2014

INFN - Laboratori Nazionali del Gran Sasso, Assergi, Italy

${ }^{*}$ Speaker. 


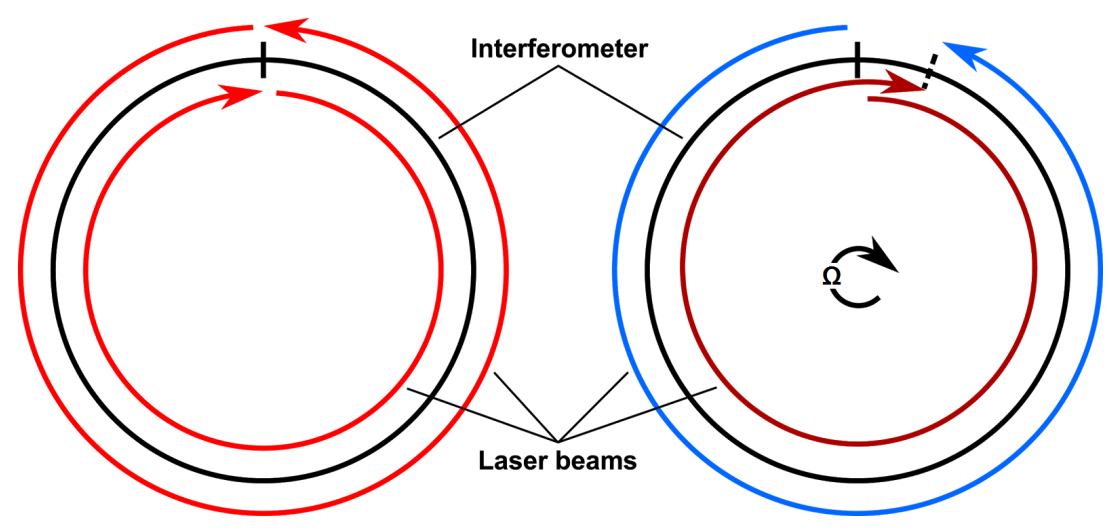

Figure 1: Simplified representation of a ring laser. When the ring does not rotate, the two laser beams travel the same distance. If the ring rotates, the two beams travel different distances.

\section{The ring laser}

Ring laser gyroscopes are nowadays the most accurate instruments to measure rotations with respect to the inertial reference frame of the universe. A ring laser converts very small time differences in measurable frequency differences. Even though the principle of operation of a ring laser is quite simple, they can reach a sensitivity as high as $1.2 \times 10^{-11} \mathrm{rad} /(\mathrm{s} \sqrt{\mathrm{Hz}})$, resolving an angular speed of about $1.5 \times 10^{-13} \mathrm{rad} / \mathrm{s}$ in 4 hours integration time [1].

The underlying principle of working of a ring laser is the Sagnac effect, which is here outlined following a classical interpretation. Let us consider an interferometer like the one shown in figure 1 , with the laser source incorporated in the cavity itself. As soon as the laser cavity is turned on, two counter propagating beams adjust themselves and give rise to a standing wave. If the ring is not rotating, both the clockwise an the anticlockwise beams travel the same distance and reach again the source point after the same time $t=\frac{2 \pi R}{c}$. If the ring itself is rotating, the two counter propagating beams do not travel the same distance. Depending on the rotation direction, one beam must travel a greater distance than the other one in order to get to the new source position. In fact, albeit the angular displacement of the ring is very small, it makes the source move along the circular path of the light. As a result, because of the invariance of the speed of light, the time taken by the two beams to reach the new source position is different.

Looking at the figure, the time taken by the counterclockwise/clockwise (+/-) beam is

$$
t_{ \pm}=\frac{2 \pi R}{c \pm \Omega R}
$$

Also $\Delta t=t_{-}-t_{+}$can be rewritten as

$$
\Delta t=\frac{4 \pi \Omega R^{2}}{c^{2}-(\Omega R)^{2}} \approx \frac{4 \pi \Omega R^{2}}{c^{2}}
$$

because $\Omega R$ is much smaller than c. This means that in the cavity can survive two standing waves with slightly different frequency $f_{ \pm}=\frac{N}{t_{ \pm}}$, giving rise to a beat. The frequency difference, known as the "Sagnac frequency", can be easily measured by deflecting part of the two beams on a photodiode and observing the beat note between them [1]. Considering the area of the ring $A=\pi R^{2}$ and its 
perimeter $P=2 \pi R$, together with the lasing condition $P=N \lambda$, where $\lambda$ is the laser wavelength, the frequency difference can be written as

$$
f_{S}=f_{+}-f_{-}=N\left(\frac{1}{t_{+}}-\frac{1}{t_{-}}\right) \approx N \frac{\Delta t}{t^{2}}=N \frac{4 \pi \Omega R^{2}}{c^{2}} \frac{c^{2}}{(2 \pi R)^{2}}=N \frac{4 A \Omega}{P^{2}}
$$

Moreover, it is possible to consider a different orientation between the ring laser and the angular speed direction by introducing the scalar product $\vec{A} \cdot \vec{\Omega}=A \Omega \cos \theta$ :

$$
f_{S}=\frac{4 \vec{A} \cdot \vec{\Omega}}{\lambda P}
$$

The quantity $S=\frac{4 A}{\lambda P}$, called "scaling factor", has the greater influence on the ring laser sensitivity. Although this relation has been obtained for a circular ring laser, it is possible to show that is valid for every shape [2].

\section{Error sources}

In the experimental practice the situation is much more complicated and there are many possible sources of error to take into account [3]. The most important sources of error are:

- room temperature and pressure variation;

- differences in the two laser beams intensities;

- backscattering.

Any variation in the room temperature and pressure can greatly affect the scaling factor. In order to avoid this problem, ring lasers have to be taken at constant temperature and pressure as much as possible. Furthermore, it is possible to actively control the positions of the mirrors to better keep the scaling factor constant [7].

Possible differences in the laser beams intensities could cause independent mode jumps of the counter-propagating beams. Such a behavior is commonly called "split mode" and cause the ring laser to be blind to rotation.

Moreover, part of the beams is inevitably backscattered by the mirrors or by some powder inside the cavity. This result in a coupling between the two counter-propagating beams (for example the clockwise and the backscattered anticlockwise) at the discharge of the laser, and the Sagnac frequency is not anymore proportional to the angular speed as indicated in equation (1.4). This problem strongly depends on the ring laser size [4].

\section{Application to seismology}

Ring lasers rigidly attached to the Earth have been able to measure the signal of the rotational component of the seismic waves for the first time [5, 6]. In seismology it is interesting to discriminate between tilts, translational displacement and rotational motion. Ring lasers are able to accurately measure the rotational motion, thus permitting a reliable evaluation of the other contributions involved in a seismic event. In particular, being a ring laser blind to any translational displacement, it is only sensitive to rotations. The frequency range of interest of the seismic rotational signals spans several orders of magnitude $\left(10^{-14} \mathrm{rad} / \mathrm{s} \leq \Omega_{s} \leq 1 \mathrm{rad} / \mathrm{s}\right)$ and large ring lasers are nowadays the only rotation sensors able to measure this kind of signal [1]. 


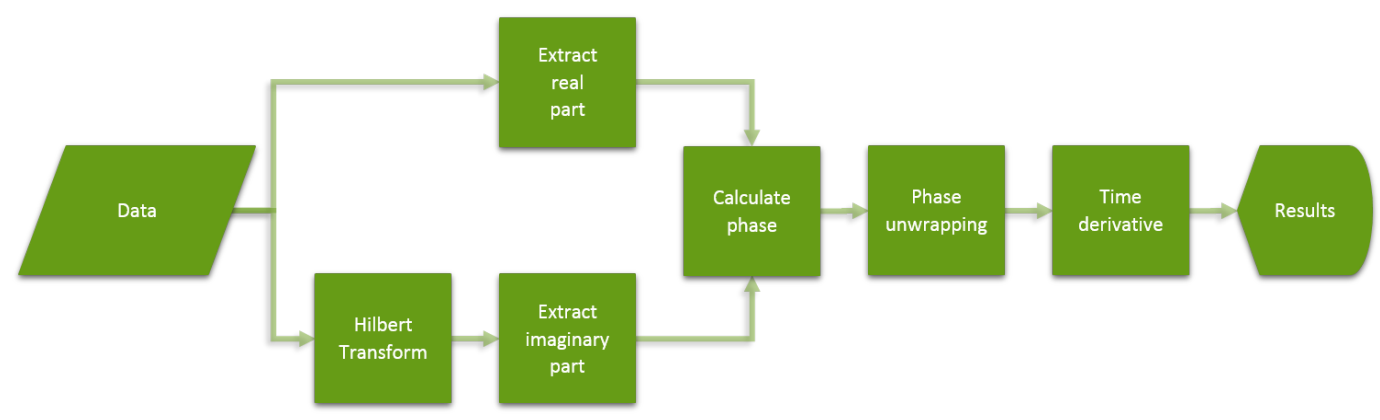

Figure 2: Data analysis procedure.

\section{The Aegean Sea earthquake of May 24, 2014}

The Aegean Sea earthquake of May 24, 2014 had a moment magnitude of 6.9 which identifies a severe event in the Mercalli scale. It took place at 09:25:03 UTC and its epicenter was between the isle of Lemnos and the isle of Samothrace $\left(40.305^{\circ} \mathrm{N} 25.453^{\circ} \mathrm{E}\right)$ with a depth of about $10 \mathrm{~km}$. The data I analyzed comes from the GP2 ring laser, located at the INFN laboratories in San Piero a Grado, close to Pisa (Italy). The GP2 ring laser is a square ring cavity with a $1.6 \mathrm{~m}$ side [7]. A detailed description of the apparatus is given in [4].

\section{Data analysis procedure}

The raw data, consisting of the voltage values coming from the photodiode, digitized by the acquisition chain, follow an analysis procedure I developed during the Gran Sasso Summer Institute 2014. This is a quite rough analysis, but contains the basics of a more precise work.

In order to analyze the data I wrote a LabVIEW code. I used LabVIEW because it provides all the tools I needed for the analysis, thus I was able to write the code in a relatively short time.

The data file contains the information on the sampling frequency and the voltage values, therefore, the data are processed by using the following procedure (as outlined in figure 3):

- the data file is read and a waveform is created and visualized in order to check for anomalies and select the data I'm interested in (sometimes the interferogram is interrupted by "split mode" intervals);

- the waveform is band-pass filtered around the Sagnac frequency (Chebyshev 1st order, 90 $\mathrm{Hz}$ bandwidth as indicated in [3]);

- the real part of the waveform and the imaginary part of its Hilbert transform are combined to form the analytic signal in polar coordinates;

- the phase is extracted from the analytic signal and unwrapped;

- the time derivative of the phase is calculated;

- the phase derivative, divided by $2 \pi$, is low-pass filtered (Butterworth $3 \mathrm{rd}$ order, $3 \mathrm{~Hz}$ cutoff frequency) and the mean is subtracted;

- the angular speed is calculated by multiplying the phase derivative by the scaling factor.

This procedure permits to choose different signal intervals and analyze them separately. Four intervals have been isolated and an independent analysis has been carried out for each one. 


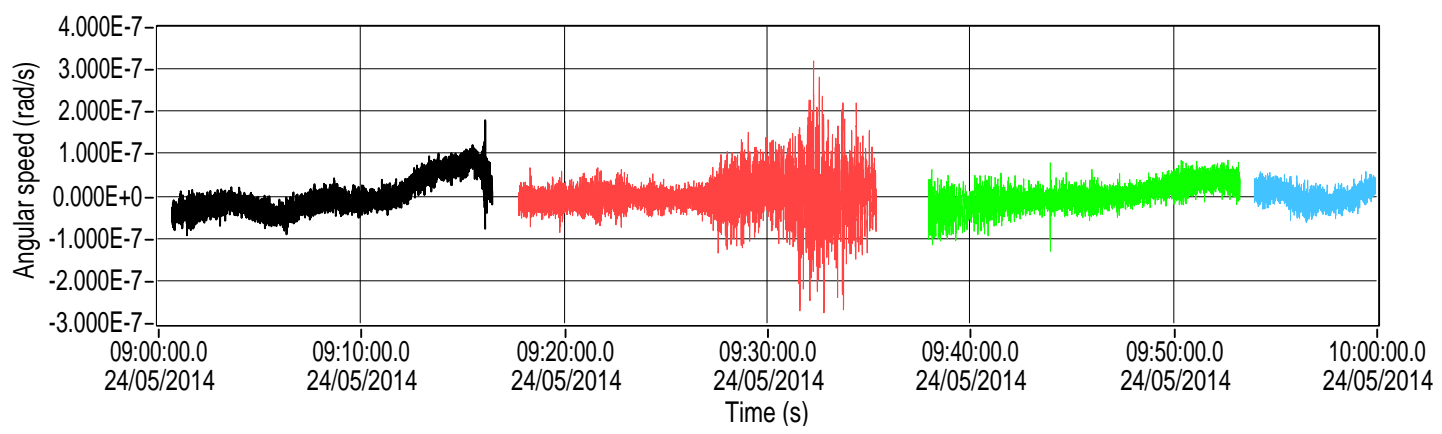

Figure 3: The signal, divided into four intervals, has been filtered and the mean has been subtracted to better evaluate the contribution of the seismic wave. In the second interval the seismic wave is clearly visible. Some long period oscillations are visible from $-1 \times 10^{-7} \mathrm{rad} / \mathrm{s}$ up to $1 \times 10^{-7} \mathrm{rad} / \mathrm{s}$ in

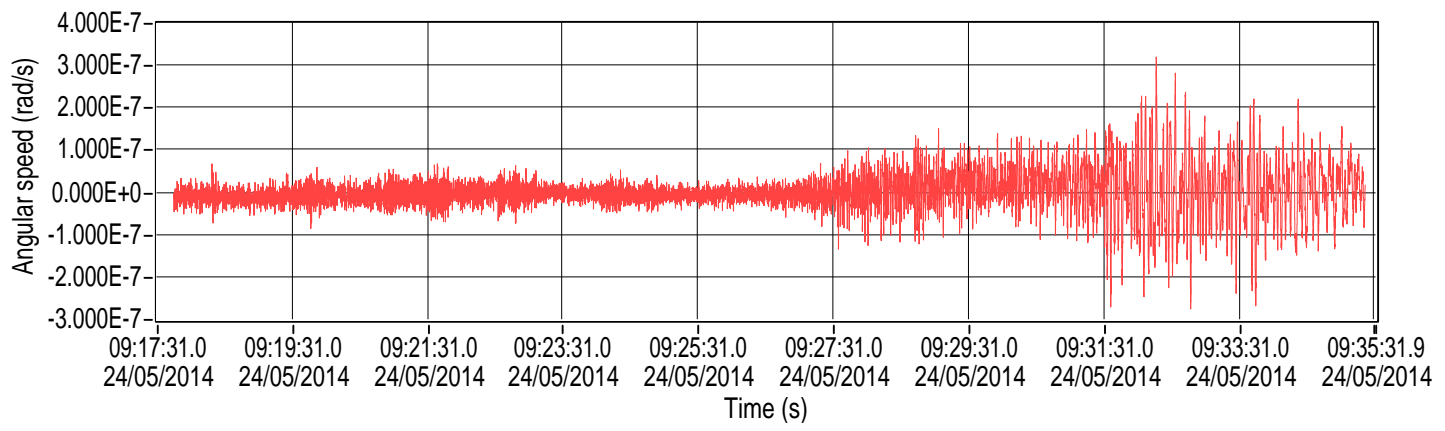

Figure 4: The oscillations caused by the seismic wave start from 9:27 UTC and range from $-3 \times 10^{-7} \mathrm{rad} / \mathrm{s}$ to $3 \times 10^{-7} \mathrm{rad} / \mathrm{s}$ in a $10 \mathrm{~s}$ long time interval.

\section{The seismic wave}

The seismic wave is visible in the second signal interval in figure 3. Setting the cutoff frequency of the low-pass filter down to $2 \mathrm{~Hz}$, the seismic wave becomes clearly distinguishable and the event time could be set around 9:27 UTC. As it is visible in figure 3, the angular speed oscillations induced by the seismic wave range from $-3 \times 10^{-7} \mathrm{rad} / \mathrm{s}$ to $3 \times 10^{-7} \mathrm{rad} / \mathrm{s}$ in a time interval of about $10 \mathrm{~s}$ (unfortunately the ring laser went in "split-mode" during the last seconds).

\section{The Earth angular speed}

After the low-pas filtering, the Earth angular speed has been evaluated for each signal interval by calculating the mean and then it has been subtracted. The obtained mean values for each interval are visible in table 3. In order to have the best value for the angular speed, it is convenient to calculate the variance weighted mean of the values obtained for each interval. This is possible under the assumption of normally distributed, independent aleatory variable with the same mean. Because of the long period oscillations, very clearly visible in figure 3 , it seems reasonable to consider a systematic uncertainty of $2 \times 10^{-7} \mathrm{rad} / \mathrm{s}$ in the evaluation of the Earth angular speed. The obtained value is

$$
\Omega_{\oplus}=\left(7.308 \pm 0.001_{\text {stat. }} \pm 0.020_{\text {sys. }}\right) \times 10^{-5} \mathrm{rad} / \mathrm{s}
$$




\begin{tabular}{cc}
\hline $\begin{array}{c}\Omega_{\oplus} \\
\left(10^{-5} \mathrm{rad} / \mathrm{s}\right)\end{array}$ & $\begin{array}{c}\text { stat. unc. } \\
\left(10^{-5} \mathrm{rad} / \mathrm{s}\right)\end{array}$ \\
\hline 7.322 & 0.003 \\
7.292 & 0.003 \\
7.311 & 0.002 \\
7.306 & 0.002 \\
\hline
\end{tabular}

Table 1: Earth angular speed calculated for each signal interval.

\section{Conclusions}

The seismic wave from the 2014 Aegean Sea earthquake has been detected and its intensity matches with the values reported in literature [4]. The angular speed of the Earth has been measured and it is in good agreement with the IERS value [8].

\section{References}

[1] K. U. Schreiber and J.-P. R. Wells, Invited Review Article: Large ring lasers for rotation sensing, Rev. Sci. Instrum. 84, 041101 (2013); doi: 10.1063/1.4798216

[2] R. D. Klauber, Derivation of the general case sagnac result using non-timeorthogonal analysis, Found. Phys. Lett. 16: 447-463 (2003).

[3] J. Belfi, N. Beverini, G. Carelli, A. Di Virgilio, E. Maccioni, G. Saccorotti, F. Stefani, A. Velikoseltsev, Horizontal rotation signals detected by "G-Pisa" ring laser for the Mw =9.0, March 2011, Japan earthquake, J. Seismol. 16: 767-776 (2012); doi: 10.1007/s10950-012-9276-9.

[4] A. Simonelli, Il giroscopio laser come sensore di rotazioni: simulazioni e attività sperimentale, Tesi di laurea magistrale, Università di Pisa, A.A. 2013/2014.

[5] D. P. McLeod, G. E. Stedman, T. H. Webb, and K. U. Schreiber, Comparison of standard and ring laser rotational seismograms, Bull. Seismol. Soc. Am. 88, 1495 (1988).

[6] A. Pancha, T. H. Webb, G. E. Stedman, D. P. McLeod, and K. U. Schreiber, Ring laser detections of rotations from teleseismic waves, Geophys. Res. Lett. 27: 3553 (2000); doi: 10.1029/2000GL011734.

[7] J. Belfi, N. Beverini, M. Calamai, G. Carelli, E. Maccioni, A. Di Virgilio, R. Santagata, A. Porzio, D. Cuccato, A. Ortolan, S. Solimeno, Absolute Control of the Scale Factor in GP2 Laser Gyroscope: Toward a Ground Based Detector of the Lense-Thirring Effect, 2013 Joint European Frequency and Time Forum \& International Frequency Control Symposium (EFTF/IFC), 795-798; doi: 10.1109/EFTF-IFC.2013.6702268.

[8] IERS Numerical Standards (IAG 1999) 